\title{
Effect of Dietary Yeast Culture Supplementation on the Growth Performance and Cecal Microbiota Modulation of Geese
}

\section{Yating Cheng}

Southwestern University https://orcid.org/0000-0002-0395-0760

\section{Kun Wan}

Southwestern University

\section{Zibiao Xiong}

Southwestern University

Hui Luo

Southwestern University

\section{Anfang Liu}

Southwestern University

\section{Hang He}

Chongqing three gorges vocational college

Jie Zhang ( $\square$ zhangjie813@163.com )

College of Animal Science, Southwest University, Chongqing 402460, China https://orcid.org/00000002-8175-1900

\section{Research Article}

Keywords: goose, yeast culture, GIT, microbiota, feed additives

Posted Date: November 5th, 2020

DOI: https://doi.org/10.21203/rs.3.rs-101816/v1

License: (c) (1) This work is licensed under a Creative Commons Attribution 4.0 International License. Read Full License 


\section{Abstract}

\section{Background}

One of the most extensively applied animal feed additives is yeast culture (YC), which can increase production efficiency by altering gastrointestinal tract (GIT) microbiota. However, its use is still limited in waterfowl. Geese are the ideal model to study the interaction between dietary and GIT, due to their adaptation to consume different roughage sources. Therefore, the effect of YC supplementation at different concentrations $(0 \%, 0.5 \%, 1.0 \%, 2.0 \%$ and $4.0 \%)$ on the GIT microbiota of geese was investigated in the present study.

Results

Three hundred Sichuan white geese with healthy and similar body weight (BW: $95.57 \pm 2.42 \mathrm{~g}$ ) were randomly divided into five groups: i) basal diet (control), ii) basal diet $+0.5 \%$ YC (treat1), iii) basal diet+1.0\% YC (treat2), iv) basal diet+2.0\% YC (treat3), and v) basal diet+4.0\% YC (treat4). After 10 weeks, slaughter and collected the cecum contents, then analysis GIT microbiota by high-throughput sequencing. The results showed that $Y C$ supplementation did not significantly affect a-diversity $(P \otimes 0.05)$. Principal coordinates analysis showed an obvious separation between control and treat4. The dominant phyla were Firmicutes and Bacteroidetes whereas the predominant genera were Alistipes and Desulfovibrionaceae. The relative abundance of Firmicutes significantly increased in the treat 1 group, whereas that of Bacteroidetes significantly decreased in the treat4 group. Dietary YC increased the proportion of beneficial bacteria, such as Parabacteroides, Enterococcus, Streptococcus and Pseudomonas, particularly in group treat2. Furthermore, treat3 significantly improved the body weight and feed utilization of geese.

\section{Conclusion}

Collectively, these findings demonstrate that dietary $Y C$ supplementation tends to increase species diversity and richness of GIT microbiota in geese. This increases the proportion of beneficial bacteria which improves amino acid and carbohydrate metabolism. Moreover, YC increases the relative abundance of Firmicutes that promote energy utilization and nutrition absorption, thereby improving the growth performance of geese. This dietary strategy based on feed additives is an effective method to maintain the health of the geese GIT and to improve growth efficiency.

\section{Introduction}

Antibiotics combat disease in animals and improve production efficiency and quality, resulting ultimately in economic profits. Therefore, antibiotics have been widely used as feed additives for animal husbandry. However, their overuse has caused many problems, such as presence of antibiotic residue, drug resistance and others ${ }^{[1]}$. Therefore, it is imperative to explore feed additives with similar effects to antibiotics, but which are safer and more effective. Yeast cultures (Ycs) are micro-ecological products 
formed by yeast after sufficient anaerobic fermentation on specific media and are mainly composed of yeast metabolites, fermented medium and a few yeast cells. YCs are rich in vitamins, saccharides, minerals, enzymes, growth-promoting factors and amino acids, which may benefit animal growth, metabolism, and health. YC is one of the most important feed additives ${ }^{[2]}$ as it can improve yield and quality of animal by-products, such as milk ${ }^{[3]}$ and eggs ${ }^{[4]}$. YC can also enhance animal growth performance, as measured by average daily gain, body weight and average daily intake ${ }^{[5-7]}$, and also can strengthen immunity and disease resistance ${ }^{[8-10]}$. The constituents of $Y C$ are live yeast and various yeast metabolites. These may act on the gastrointestinal tract (GIT) directly to maintain the balance of the microorganism and promote development of the GIT. For example, YC can significantly affect community structure and composition of gut microbiota of grass carp, increasing the abundance of beneficial bacteria ${ }^{[11]}$. Addition of $Y C$ in the diet resulted in an increase in beneficial bacteria, making greater compensatory weight gains in weanling pigs ${ }^{[12]}$. The effect of $Y C$ on hindgut microbial communities was evaluated in horse ${ }^{[13]}$ where it increased the abundance of fiber-degrading bacteria. Geese are important poultry in China ( $90 \%$ of the world's production), and their cecum are more developed compared with other poultry, so it is the ideal model to study the interaction between GIT microbiota and diet ${ }^{[14]}$. Supplementation of geese diets with yeast fermanted feed regulate cecal microflora that is beneficial of growth performance ${ }^{[15]}$. Addition of yeast supplement in the diets improve intestinal microflora of goose and lead to better carcass hygiene ${ }^{[16]}$. However, few studies have investigated the effect of YC on the GIT microbiota of geese. Furthermore, the variety in GIT microbiota composition and structure can reflect the effect of dietary on the host. Thus, the present study was conducted to investigate the effect of YC supplementation on the GIT microbiota of geese.

\section{Methods}

\section{Animals, diets, feeding and experimental design}

Three hundred Sichuan white geese with healthy and similar body weight (BW: $95.57 \pm 2.42 \mathrm{~g}$ ) were randomly divided into five groups: i) basal diet (control), ii) basal diet $+0.5 \% \mathrm{YC}$ (treat1), iii) basal diet + $1.0 \%$ YC (treat2), iv) basal diet $+2.0 \%$ YC (treat3), and v) basal diet $+4.0 \%$ YC (treat4). The commercial YC (Baihuibang, Beijing Enhalor Biotechnology Co., Ltd., Beijing, China) was a fermented product composed of Saccharomyces cerevisiae grown on a medium, which contains 15.0\% CP, 3.5\% crude fat, $~ 8.7 \%$ crude fiber, $~ 14.2 \%$ amino acid, 3.3\% mannan, 14.0\% $\beta$-glucan and other micro-components. Each group was fed four times a day (at 7:30, 12:30, 17:00 and 21:00) for 10 weeks. Two corn-soybean based basal diets were formulated to be fed during starter ( $0-4$ weeks) and grower ( $5-10$ weeks; Table 1 ) periods. Each group consisted of 3 replicates pens with 20 geese per pen. Geese were allowed access to feed (granule form) and water ad libitum throughout the experimental period. The daily temperature inside the house throughout the experimental period was $(21.32 \pm 2.39)^{\circ} \mathrm{C}$, and the relative humidity was $(84.03 \pm 5.15) \%$. 
Table 1

The composition of the basal diet of geese.

\begin{tabular}{|c|c|c|}
\hline Items & $0-4$ weeks & $5-10$ weeks \\
\hline \multicolumn{3}{|l|}{ Ingredients (\%) } \\
\hline Corn & 63.80 & 53.60 \\
\hline Wheat bran & 2.99 & 14.50 \\
\hline Soybean meal & 20.00 & 11.50 \\
\hline Rapeseed meal & 4.00 & / \\
\hline Rice bran & / & 13.40 \\
\hline Silkworm chrysalis & 4.30 & 1.79 \\
\hline Calcium hydrogen phosphate & 1.59 & 0.90 \\
\hline Stone powder & 0.87 & 0.75 \\
\hline Salt & 0.20 & 0.20 \\
\hline L-Lysine (98\%) & 0.15 & 0.18 \\
\hline DL-methionine & 0.05 & 0.07 \\
\hline Choline chloride & 0.05 & 0.12 \\
\hline Premix & 2.00 & 2.00 \\
\hline Sand & / & 1.00 \\
\hline Total & 100 & 100 \\
\hline \multicolumn{3}{|l|}{ Nutrient content } \\
\hline Metabolizable Energy $\left(\mathrm{MJ} \cdot \mathrm{kg}^{-1}\right)$ & 11.97 & 11.21 \\
\hline Crude protein (\%) & 20.43 & 14.81 \\
\hline Crude fiber (\%) & 4.12 & 8.04 \\
\hline Calcium (\%) & 0.87 & 0.80 \\
\hline Available P (\%) & 0.43 & 0.40 \\
\hline Lysine (\%) & 1.14 & 0.85 \\
\hline Methionine (\%) & 0.36 & 0.30 \\
\hline \multicolumn{3}{|c|}{$\begin{array}{l}\text { Note: Premix provides (per kilogram of diet): vitamin A } 2000 \text { IU, vitamin D } 3350 \mathrm{IU} \text {, vitamin E } 3000 \mathrm{mg} \\
\text { vitamin K } 3200 \mathrm{mg} \text {, vitamin B1 } 100 \mathrm{mg} \text {, vitamin B21 } 200 \mathrm{mg} \text {, vitamin B6 } 200 \mathrm{mg} \text {, vitamin B12 } 2.5 \mathrm{mg} \\
\text { niacin } 6000 \mathrm{mg} \text {, pantothenic acid } 200 \mathrm{mg} \text {, folic acid } 200 \mathrm{mg} \text {, biotin } 20 \mathrm{mg} \text {, choline } 7.0 \mathrm{~g} \text {, iron } 6 \mathrm{~g} \text {, } \\
\text { copper } 0.2 \mathrm{~g} \text {, manganese } 15 \mathrm{~g} \text {, zinc } 8 \mathrm{~g} \text {, iodine } 10 \mathrm{mg} \text {, selenium } 30 \mathrm{mg} \text {. }\end{array}$} \\
\hline
\end{tabular}




\section{Growth performance measurements}

The BW was measured on days 1 and 70 with empty stomach, and feed consumption was recorded every morning before the feeding. Average daily gain (ADG), average daily feed intake (ADFI) and feed conversion ratio (FCR) were calculated for the total period.

\section{Intestinal content collection}

Three 70 day old geese were randomly selected from each pen and killed. Nine cecum contents samples from each group were extracted and pooled by $3(n=3)$. The cecum was dissected with sterile instruments and washed in $70 \%$ ethanol and sterile water to avoid transient bacteria. The content from cecum was squeezed out and mix thoroughly, then collected into sterilized tubes and immediately stored in liquid nitrogen.

\section{S rRNA PCR amplification and sequencing}

Bacterial genomic DNA was extracted from $100 \mathrm{mg}$ of each sample using the QIAamp DNA Stool Mini Kit (Qiagen, Germany) following the manufacturer's protocol. The bacterial V3-V4 region of the 16S rRNA was amplified using the following primers: 319F (5'-ACTCCTACGGGAGGCAGCAG-3') and 806R (5'GGACTACHVGGGTWTC TAAT-3'), and a sequencing adapter was added to the end of the primers. Then the PCR products were purified, quantified, and homogenized to establish a sequencing library. The constructed library was subjected to library quality inspection, and the qualified library was sequenced as described in Fadrosh, Ma, Gajer, Sengamalay, Ott, Brotman and Ravel ${ }^{[17]}$ using Illumina HiSeq 2500 (Illumina, San Diego, CA, USA). All control and YC-treated samples were included in the same sequencing run.

\section{Sequences analysis}

According to the overlap relationship between paired-end (PE) reads, the double-end sequences obtained from Hi-seq sequencing were merged into a single sequence tag, to control the quality of reads and filter the effect of merge. This included the following three main steps: 1) PE reads merging: used FLASH v1.2.7 to merge the reads of each sample through the overlap, obtaining the original tags data (raw tags); 2) tags filtering: used Trimmomatic v0.33 to filter the raw tags obtained by merging to obtain high quality tags data (clean tags); 3 ) chimera removal: used UCHIME v4.2 to identify and remove the chimera sequence to obtain the final effective data (effective tags).

\section{Bioinformatics analysis}

Usearch ${ }^{[18]}$ was used to cluster tags at a similarity level of $97 \%$ to identify operational taxonomic units (OTUs). OTUs were annotated based on the Silva (bacterial) and UNITE (fungi) taxonomy databases. Taxonomic assignment was achieved using RDP classifier, clustering the sequences at $97 \%$ similarity with a confidence threshold of 0.8 .

\section{Microbiota diversity analysis}


The $\alpha$ - and $\beta$-diversity of each sample was evaluated based on the OTU data (Supplementary Material S1). Mothur (version v.1.30) and QIIME were used to calculate $\alpha$ - and $\beta$-diversity, respectively. PICRUSt ${ }^{\text {[19] }}$ was used to detect the composition of functional genetic of each group by comparing species information and analyzing differences between groups in KEGG Orthologs (KO) composition including function. This involved metabolism, genetic information processing, human diseases, environmental information processing, cellular processes and organismal systems. Bugbase was used to detect the potential pathogenic bacteria abundance at the phylum and genus level.

\section{Statistical analysis}

The data analyses were performed with SPSS 22.0 software (IBM Corporation, 2014) using one-way ANOVA with LSD multiple comparison tests. All data are shown as means \pm SEM unless otherwise indicated. Differences were considered statistically significant when $P<0.05$ throughout.

\section{Results}

\section{Sequencing data analysis}

A total of 1,195,635 couples of effective reads were obtained from $16 \mathrm{~S}$ rRNA gene V3 + V4 amplicons with an average of 79,709 reads per sample (ranging from 76,541 to 80,376 ). Detailed data is shown in Supplementary Table S1. At a similarity level of $97 \%$, we found a total of 484 OTUs common to all groups, where the number of OTUs in different groups (Fig. 1a) is: control, 477; treat1, 479; treat2, 481; treat3, 480; treat4, 475). A heat map with the 50 most abundant bacteria at the family level in the five groups (Fig. 1b) suggests that bacterial abundance in the control was different from the YC groups. A total of 462 common OTUs were shared among the five groups, and the most abundant taxa of the shared OTUs (Fig. 1c) indicate that Firmicutes, Bacteroidetes, Proteobacteria, Acidobacteria, Verrucomicrobia and Cyanobacteria form the six most dominant phyla. Seven unique OTUs were found in the YC-group compared with the control (Fig. 1d). Among them, four OTUs at the genus level, Butyrivibrio, Ruminococcus_1, DTU014, and Bacteroides were found in the YC-group, Caproiciproducens and Clostridiales_vadinBB60_group were found in the YC-group except treat1, and [Eubacterium] coprostanoligenes_group was found in the treat3 and trea4.

\section{Variation in microbiota diversity}

The addition of $Y C$ in the diet produced no significant differences $(P>0.05)$ in a-diversity, as measured using Shannon, Simpson, Ace and Chao1 indices (Table 2). Using the Simpson's diversity index, the sequence is the same but in opposite order. These results indicate that the diet supplemented with $0.5 \%$ and $4 \%$ of YC caused the highest and the lowest species diversity, respectively. The Chao1 and Ace indices indicating that the diets supplemented with $2 \%$ and $4 \% \mathrm{YC}$ had the highest and lowest microbial richness, respectively. The $\beta$-diversity, based on the overall community composition, indicated that the similarity between clustered GIT microbiota in the five groups was due to the YC supplement (Fig. 2). This figure shows that group treat4 clearly separated from the other groups, using both weighted and 
unweighted PCoA analysis, whereas treat 1 and treat 3 formed a cluster. In addition, the replicates in treat 2 show a large variation. These results are significant since the sum of PC1 and PC2 was more than 50\%, therefore these results likely represent the differences in GIT microbial community structure among these five groups.

Table 2

Effect of dietary yeast culture supplementation on the gut microbiota a-diversity of geese.

\begin{tabular}{|llllll|}
\hline Item & control & treat1 & treat2 & treat3 & treat4 \\
\hline Shannon & $3.48 \pm 0.29$ & $3.83 \pm 0.18$ & $3.55 \pm 0.34$ & $3.56 \pm 0.35$ & $2.84 \pm 0.58$ \\
\hline Simpson & $0.144 \pm 0.013$ & $0.129 \pm 0.005$ & $0.136 \pm 0.006$ & $0.130 \pm 0.021$ & $0.156 \pm 0.033$ \\
Ace & $460.85 \pm 12.71$ & $466.61 \pm 4.31$ & $467.45 \pm 2.25$ & $469.30 \pm 5.53$ & $450.53 \pm 8.53$ \\
Chao1 & $463.55 \pm 13.23$ & $467.44 \pm 6.84$ & $469.58 \pm 21.16$ & $470.85 \pm 9.17$ & $452.74 \pm 7.37$ \\
All data represent means \pm SEM. & & & \\
\hline
\end{tabular}

\section{Microbial analysis at the phylum and genus level}

At the phylum level, a total of 13 phyla were identified (control: 13; treat1: 12; treat2: 11; treat3: 13; treat4: 12). Firmicutes (19.90 54.60\%), Bacteroidetes (21.60 59.20\%), Proteobacteria (8.09 23.50\%), Actinobacteria (1.77 4.65\%), Cyanobacteria (1.01 2.52\%) and Verrucomicrobia (0.55 5.46\%) constituted the dominant phyla (Fig. 3a). At the genus level, a total of 189 genera were identified (control: 186; treat1: 188; treat2: 189; treat3: 187; treat4: 184). Alistipes (17.6 49.7\%), Desulfovibrionaceae (4.57\% 18.20\%), Lachnospiraceae (1.49\% 7.64\%), CHKCI001 (1.23\% 14.10\%), Blautia (1.42\% 5.30\%), Bacteroides (2.30\% 8.24\%) were predominant (Fig. 3b).

\section{Comparison of the relative abundance of bacteria}

YC treatment produced a significant effect on the relative abundance at both phylum and genus levels (Fig. 4). Compared with the control, the relative abundance of phyla Bacteroidetes and Firmicutes in treat4 significantly increased or decreased, respectively $(P<0.05)$, whereas Firmicutes significantly increased in treat1 treat2 and treat3 $(P<0.05)$. In treat1, the relative abundance of genus Shuttleworthia, Christensenellaceae_R-7_group, Ruminococcaceae_UCG-005, Faecalibacterium, Eisenbergiella, Blautia and Sellimonas significantly increased $(P<0.05)$, whereas in treat 4 , genus Alistipes and Bacteroides also increased $(P<0.05)$.

\section{Predicted functions of microbiota}

Based on functionality prediction, we investigated the $\mathrm{KO}$ composition in the five groups. We found no significant differences between control, treat2, treat3 and treat4. However, neurodegenerative diseases, substance dependence, cancers: specific types, carbohydrate metabolism, amino acid metabolism, global 
and overview maps, aging and sensory system achieve a statistical significant difference $(P<0.05)$ between control and treat1 (Fig. 5a). To determine whether the YC supplementation has a positive or negative effect on the GIT, and what levels are optimal, we predicted what bacteria were pathogenic and beneficial in the five groups (Fig. 5b). At the genus level, the proportion of potential beneficial bacteria in treat2 was higher than in other groups, whereas the proportion of potential pathogenic bacteria in treat4 was higher than in other groups. Among these bacteria, the dominant potential pathogenic genera were Rikenellaceae, Bacteroides and Ruminococcus, whereas the prominent potential beneficial genera were Parabacteroides, Enterococcus, Streptococcus and Pseudomonas.

\section{Growth performance}

There was no significant difference in the initial BW of geese with different $Y C$ dietary groups. Dietary YC supplementation significantly affected the final BW, ADG, ADFI, and F/G (Table 3). Compared with the control, the $Y C$ dietary significantly increased the final $B W$ and $A D G$, while significantly decreased the $F / G$, with treat3 being the most effective. Moreover, ADFI in treat3 was significantly reduced, and no significant differences were found among other groups.

Table 3

Effect of dietary yeast culture supplementation on growth performance of geese.

\begin{tabular}{|c|c|c|c|c|c|}
\hline Item & control & treat1 & treat2 & treat3 & treat4 \\
\hline $\begin{array}{l}\text { Initial BW } \\
\text { (g) }\end{array}$ & $94.50 \pm 1.32$ & $96.33 \pm 0.76$ & $94.67 \pm 3.06$ & $95.67 \pm 3.06$ & $96.67 \pm 4.16$ \\
\hline $\begin{array}{l}\text { Final BW } \\
\text { (g) }\end{array}$ & $\begin{array}{l}2868.00 \pm \\
49.15^{c}\end{array}$ & $\begin{array}{l}3024.67 \pm \\
157.03^{b}\end{array}$ & $\begin{array}{l}3026.67 \pm \\
66.01^{b}\end{array}$ & $\begin{array}{l}3474.00 \pm \\
12.17^{a}\end{array}$ & $\begin{array}{l}3063.33 \pm \\
36.02^{b}\end{array}$ \\
\hline ADG (g) & $39.62 \pm 0.68^{c}$ & $41.83 \pm 2.24^{b}$ & $41.89 \pm 0.90^{b}$ & $48.26 \pm 0.17^{a}$ & $42.38 \pm 0.46^{b}$ \\
\hline ADFI (g) & $\begin{array}{l}157.25 \pm \\
1.36^{\mathrm{a}}\end{array}$ & $156.19 \pm 0.43^{a}$ & $156.51 \pm 2.09^{a}$ & $148.01 \pm 1.71^{b}$ & $157.16 \pm 1.41^{\circ}$ \\
\hline FCR & $3.97 \pm 0.08^{a}$ & $3.74 \pm 0.21^{b}$ & $3.74 \pm 0.11^{b}$ & $3.07 \pm 0.04^{c}$ & $3.71 \pm 0.02^{b}$ \\
\hline \multicolumn{6}{|c|}{$\begin{array}{l}\text { All data represent means } \pm \text { SEM. In the same row, means with different letters are significantly } \\
\text { different }(P<0.05) \text {, means with the same letters are not significantly different }(P>0.05) \text {. Absence of } \\
\text { letters indicates no significant difference between treatments. }\end{array}$} \\
\hline
\end{tabular}

\section{Discussion}

The GIT microbiota is a community of microorganisms inhabiting the animal gastrointestinal tract, which can be beneficial or harmful. In general, there is a balance between beneficial and harmful microbiota, and this has relevance for the immune system, metabolism ${ }^{[20]}$ and diseases ${ }^{[21]}$. The composition of the GIT microbiota evolves throughout an individual's lifetime, and the balance may be altered by both 
endogenous and exogenous factors ${ }^{[22]}$, such as the diet ${ }^{[23]}$. Thus, the GIT microbial composition depends on the interaction between the microorganisms and their host, and with the diet of the host. Therefore, studying the GIT microbiota composition and its structure can be used to better understand how dietary $Y C$ affects growth performance of goose at the microbial level. In the present study, we chose five commonly used YC diet supplementation levels $(0,0.5,1.0,2.0$, and $4.0 \%)$ to study how YC modulates GIT microbiota. We used high-throughput sequencing of the V3 + V4 region of the 16S rRNA gene to explore the diversity and composition of GIT microbiota in geese.

The a-diversity analysis showed that there is no significant differences in species diversity or richness between these treatments, whereas $\beta$-diversity analysis based on PCoA showed a clear separate microbiota structure among the groups. Further, the a-diversity analysis suggested that some microorganisms are essential for the growth of the host. In addition, the presence or absence of microorganisms is not easily affected by the rearing environment or changes in diet, although the relative abundance may be affected. This is consistent with a report indicating that there are no significant differences in a-diversity when gilthead sea bream is supplemented with different mannanoligosaccharide levels ${ }^{[24]}$. However, laying hens with the higher fermentation yeast addition into diets had the lower total bacterial count ${ }^{[25]}$. The discrepancies between these and the present study may be caused by differences in yeast product used, host species and rearing conditions.

In the present study, there were clear differences in microbial composition between the different $Y C$ treatments. Firmicutes and Bacteroidetes were the two most dominant phyla in the bacterial communities of the goose. Similar results have been observed in chicken ${ }^{[26]}$, buffalo ${ }^{[27]}$ and goose ${ }^{[28]}$, where the predominant genera were Alistipes and Desulfovibrionaceae. Phylum Firmicutes and Bacteroidetes are beneficial to the host. Firmicutes are tightly related to nutrition and to energy absorption ${ }^{[29]}$. In particular, the genera of this phylum degrade fiber and cannot stand extremely acidic environments. In contrast, phylum Bacteroidetes plays a role in maintaining a healthy GIT, adapting to changing and extreme environments ${ }^{[30]}$. Furthermore, we found that as the amount of $Y C$ increased, the relative abundance of the phyla Firmicutes and Bacteroidetes decreased and increased, respectively, and a similar trend can be found in their respective genera (Fig. 3). These results are consistent with similar studies performed on gut microbiota in humans ${ }^{[31]}$. These results may be explained by considering that $\mathrm{YC}$ diet supplementation can enhance microbiota fermentation, resulting in an increase in the concentration of volatile fatty acids and a reduced $\mathrm{pH}$ of the GIT ${ }^{[32]}$. In contrast to Firmicutes, Bacteroidetes can adapt to this acidic environment.

The dominant bacteria in the GIT may play many important functions essential to the goose ${ }^{[33]}$. Our study indicates that $0.5 \%$ YC can significantly enhance carbohydrate and amino acid metabolism and global and overview maps (Fig. 5a). These biochemical processes might promote utilization of energy, absorption of nutrients and growth ${ }^{[34]}$. Remarkably, the proportion of potential beneficial bacteria after 1.0 and $2.0 \% \mathrm{YC}$ treatment was higher than in the control (Fig. 5b). These results are caused by the presence of oligosaccharides in YC, which can minimize GIT pathogen concentration and stimulate the 
growth of beneficial bacteria ${ }^{[35]}$, thus alleviating the competition for diet between the host and the pathogenic bacteria. However, the proportion of potential beneficial bacteria in the sample treated with the highest amount of YC (treat4) was the lowest (Fig. 5b). High concentration of YC may induce the goose to develop immune tolerance, leading to wastage of energy and nutrients and suppressing the growth of both host and microbiota ${ }^{[36]}$. A similar result was obtained for growth performance, where low YC concentration $(0.5 \%, 1 \%$ and $2 \%)$ improved average daily gain and feed conversion rate in goose, whereas high YC concentration (4\%) did not.

In conclusion, dietary $Y C$ modulates the composition and relative abundance of GIT microbiota in goose and lead to better growth performance. In general, dietary YC is beneficial to GIT microbiota and its effect depends on concentration. Specifically, $0.5 \%$ YC increased species diversity, $1 \%$ YC increased the proportion of beneficial bacteria and $2 \%$ YC improved species richness.

\section{Abbreviations}

YC

yeast culture

GIT

gastrointestinal tract

\section{Declarations}

\section{Ethics approval and consent to participate}

Not applicable.

\section{Consent for publication}

Not applicable.

\section{Competing interests}

The authors declare that they have no competing interests.

\section{Availability of data and materials}

The authors declare that the data of this research are not deposited in any official repository. All sequences have been deposited in the NCBI's Sequence Read Archive with the accession number PRJNA616162.

\section{Funding}

This study was financially supported by the Fundamental Research Funds for the Central Universities (XDJK2019C094). 
Acknowledgements

The authors sincerely thank Y. Z. Han (Associate Professor, Southwest University, Chongqing, China) for the revision of the manuscript.

\section{References}

[1]Baquero F, Martinez JL, Canton R. Antibiotics and antibiotic resistance in water environments. Curr Opin Biotechnol. 2008;19:260-265.

[2]Denev S, Tzonka P, Radulova P, Stancheva N, Staykova G, Beev G, Todorova P, Tchobanova S. Yeast Cultures in Ruminant Nutrition. Bulgarian Journal of Agricultural Science. 2007;13:357-374.

[3]Poppy GD, Rabiee AR, Lean IJ, Sanchez WK, Dorton KL, Morley PS. A meta-analysis of the effects of feeding yeast culture produced by anaerobic fermentation of Saccharomyces cerevisiae on milk production of lactating dairy cows. J Dairy Sci. 2012;95:6027-6041.

[4]Özsoy B, Karadağoğlu Ö, Yakan A, Önk K, Çelik E, Şahin T. The role of yeast culture (Saccharomyces cerevisiae) on performance, egg yolk fatty acid composition, and fecal microflora of laying hens. Revista Brasileira de Zootecnia. 2018;47.

[5]Van Der Peet-Schwering CM, Jansman AJ, Smidt H, Yoon I. Effects of yeast culture on performance, gut integrity, and blood cell composition of weanling pigs. J Anim Sci. 2007;85:3099-3109.

[6]Chaucheyrasdurand F, Walker ND, Bach A. Effects of active dry yeasts on the rumen microbial ecosystem: Past, present and future. Anim Feed Sci Technol. 2008;145:5-26.

[7]Shen YB, Piao X, Kim SW, Wang L, Liu P, Yoon I, Zhen YG. Effects of yeast culture supplementation on growth performance, intestinal health, and immune response of nursery pigs. J Anim Sci. 2009;87:26142624.

[8]Davis ME, Brown DC, Maxwell CV, Johnson ZB, Kegley EB, Dvorak RA. Effect of phosphorylated mannans and pharmacological additions of zinc oxide on growth and immunocompetence of weanling pigs. J Anim Sci. 2004;82:581-587.

[9]Gao J, Zhang HJ, Yu SH, Wu SG, Yoon I, Quigley J, Gao YP, Qi GH. Effects of Yeast Culture in Broiler Diets on Performance and Immunomodulatory Functions. Poultry Science. 2008;87:1377-1384.

[10]Jensen GS, Patterson K, Yoon I. Yeast culture has anti-inflammatory effects and specifically activates NK cells. Comparative Immunology Microbiology and Infectious Diseases. 2008;31:487-500.

[11]Liu H, Li J, Guo X, Liang Y, Wang W. Yeast culture dietary supplementation modulates gut microbiota, growth and biochemical parameters of grass carp. Microbial Biotechnology. 2018;11:551-565. 
[12]Price K, Totty H, Lee H, Utt M, Fitzner G, Yoon I, Ponder M, Escobar J. Use of Saccharomyces cerevisiae fermentation product on growth performance and microbiota of weaned pigs during Salmonella infection. J Anim Sci. 2010;88:3896-3908.

[13]Jouany JP, Medina B, Bertin G, Julliand V. Effect of live yeast culture supplementation on hindgut microbial communities and their polysaccharidase and glycoside hydrolase activities in horses fed a high-fiber or high-starch diet. J Anim Sci. 2009;87:2844-2852.

[14]Liu G, Luo X, Zhao X, Zhang A, Jiang N, Yang L, Huang M, Xu L, Ding L, Li M. Gut microbiota correlates with fiber and apparent nutrients digestion in goose. Poultry Science. 2018;97:3899-3909.

[15]Yan J, Zhou B, Xi Y, Huan H, Li M, Yu J, Zhu H, Dai Z, Ying S, Zhou W, Shi Z. Fermented feed regulates growth performance and the cecal microbiota community in geese. Poultry Science. 2019;98:4673-4684.

[16]Yaman H, Ulukanli Z, Elmali M, Unal Y. The effect of a fermented probiotic, the kefir, on intestinal flora of poultry domesticated geese (Anser anser). Journal of Materials Chemistry. 2006;19:1635-1642.

[17]Fadrosh DW, Ma B, Gajer P, Sengamalay N, Ott S, Brotman RM, Ravel J. An improved dual-indexing approach for multiplexed 16S rRNA gene sequencing on the lllumina MiSeq platform. Microbiome. 2014;2:6-6.

[18]Edgar RC. UPARSE: highly accurate OTU sequences from microbial amplicon reads. Nat Methods. 2013;10:996-998.

[19]Parks DH, Tyson GW, Hugenholtz P, Beiko RG. STAMP: Statistical analysis of taxonomic and functional profiles. Bioinformatics. 2014;30:3123-3124.

[20]Tremaroli V, Backhed F. Functional interactions between the gut microbiota and host metabolism. Nature. 2012;489:242-249.

[21]Pennisi E. Cancer Therapies Use a Little Help From Microbial Friends. Science. 2013;342:921-921.

[22]Sekirov I, Russell SL, Antunes LCM, Finlay BB. Gut Microbiota in Health and Disease. Physiol Rev. 2010;90:859-904.

[23]Scott KP, Gratz SW, Sheridan PO, Flint HJ, Duncan SH. The influence of diet on the gut microbiota. Pharmacol Res. 2013;69:52-60.

[24]Dimitroglou A, Merrifield DL, Spring P, Sweetman J, Moate R, Davies SJ. Effects of mannan oligosaccharide (MOS) supplementation on growth performance, feed utilisation, intestinal histology and gut microbiota of gilthead sea bream (Sparus aurata). Aquaculture. 2010;300:182-188.

[25]Hassanein S, Soliman N. Effect of Probiotic (Saccharomyces cerevisiae) Adding to Diets on Intestinal Microflora and Performance of Hy-Line Layers Hens. Journal of American Science. 2010;6. 
[26]Qu A, Brulc JM, Wilson MK, Law BF, Theoret JR, Joens LA, Konkel ME, Angly FE, Dinsdale EA, Edwards R. Comparative Metagenomics Reveals Host Specific Metavirulomes and Horizontal Gene Transfer Elements in the Chicken Cecum Microbiome. PLOS ONE. 2008;3.

[27]Singh KM, Ahir VB, Tripathi AK, Ramani UV, Sajnani MR, Koringa PG, Jakhesara SJ, Pandya PR, Rank DN, Murty DS. Metagenomic analysis of Surti buffalo ( Bubalus bubalis ) rumen: a preliminary study. Mol Biol Rep. 2012;39:4841-4848.

[28]Wang W, Cao J, Li J-R, Yang F, Li Z, Li L-X. Comparative analysis of the gastrointestinal microbial communities of bar-headed goose (Anser indicus) in different breeding patterns by high-throughput sequencing. Microbiol Res. 2015;182.

[29]Jumpertz R, Le DS, Turnbaugh PJ, Trinidad C, Bogardus C, Gordon JI, Krakoff J. Energy-balance studies reveal associations between gut microbes, caloric load, and nutrient absorption in humans. The American journal of clinical nutrition. 2011;94:58-65.

[30]Thomas F, Hehemann J, Rebuffet E, Czjzek M, Michel G. Environmental and Gut Bacteroidetes: The Food Connection. Frontiers in Microbiology. 2011;2:93-93.

[31]David LA, Maurice CF, Carmody RN, Gootenberg DB, Button JE, Wolfe BE, Ling AV, Devlin AS, Varma Y, Fischbach MA. Diet rapidly and reproducibly alters the human gut microbiome. Nature. 2014;505:559563.

[32]Millerwebster T, Hoover WH, Holt M, Nocek JE. Influence of Yeast Culture on Ruminal Microbial Metabolism in Continuous Culture. J Dairy Sci. 2002;85:2009-2014.

[33]Nicholson JK, Holmes E, Kinross J, Burcelin R, Gibson GR, Jia W, Pettersson S. Host-Gut Microbiota Metabolic Interactions. Science. 2012;336:1262-1267.

[34]Li M, Zhou H, Pan X, Xu T, Zhang Z, Zi X, Jiang Y. Correction: Corrigendum: Cassava foliage affects the microbial diversity of Chinese indigenous geese caecum using $16 \mathrm{~S}$ rRNA sequencing. Scientific Reports. 2017;7:1-1.

[35]De Maesschalck C, Eeckhaut V, Maertens L, De Lange L, Marchal L, Nezer C, De Baere S, Croubels S, Daube G, Dewulf J. Effects of Xylo-Oligosaccharides on Broiler Chicken Performance and Microbiota. Appl Environ Microbiol. 2015;81:5880-5888.

[36]Zhen YG, Zhao W, Chen X, Li LJ, Lee HG, Zhang XF, Wang T. Effects of yeast culture on broiler growth performance, nutrient digestibility and caecal microbiota. S Afr J Anim Sci. 2019;49:99-108.

\section{Figures}


a)

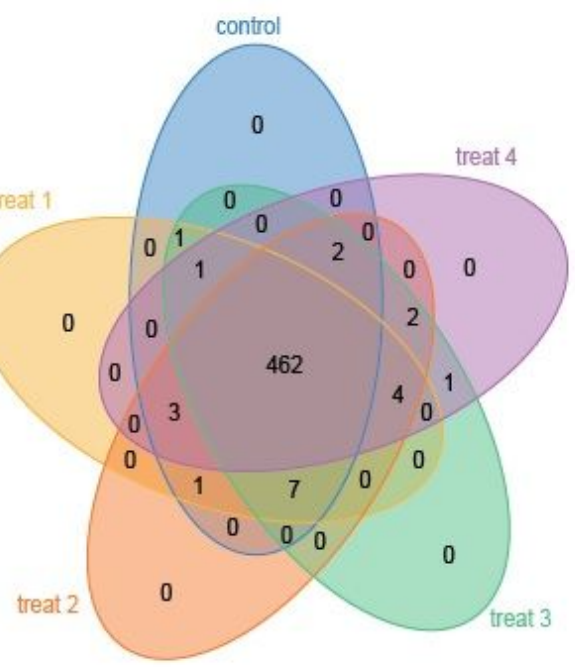

c)

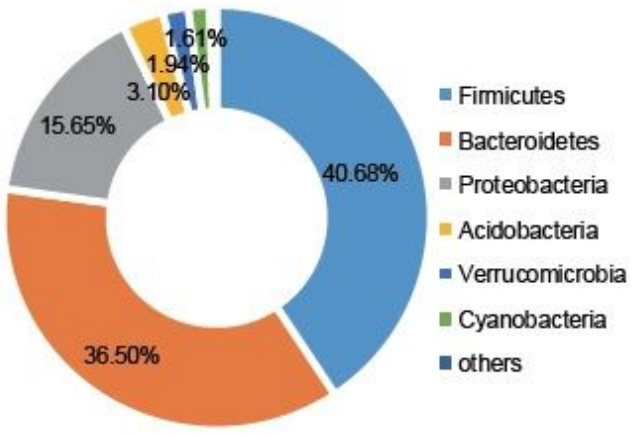

d)

口control otreat1 Otreat2 etreat3 Otreat4

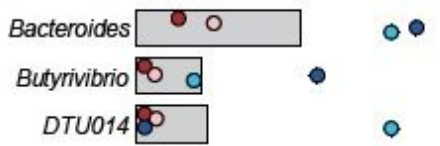

Clostridiales_vadin $B B 60$ group $\mathrm{O}_{0}$

Caproiciproducens 8

Eubacterium]_coprostanoligenes

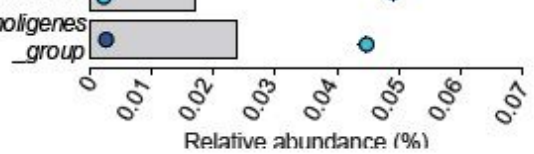

Relative ahundance (\%) b)

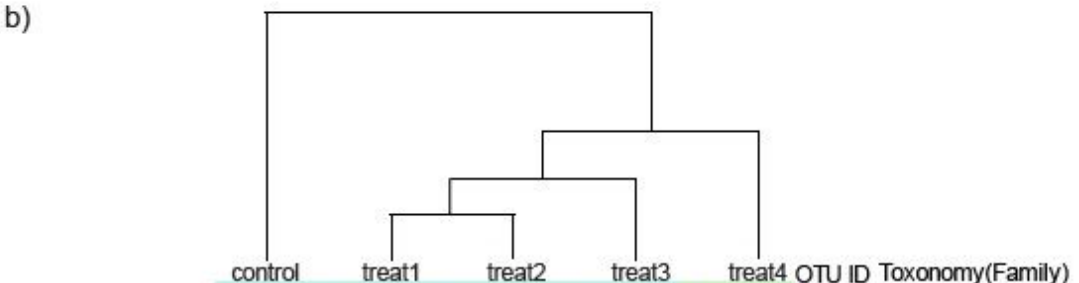

OTU ID Toxonomy(ram

OTI2 Rikenellaceae OTU23 Ruminococcaceae OTU251Rikenellaceae OTU6 Bacteroidaceae OTU7 Rikenellaceae OTU42 Bamesiellaceae OTU18 Lachnospiraceae OTU25 Ruminococcaceae OTU44 Mitochondria OTU15 Pseudomonadaceae OTU16 Uncultured OTU8 Nocardiaceae OTU17 Staphylococcaceae OTU26 Lachnospiraceae OTU30 Lachnospiraceae oTu4 Akkermansiaceae 0TU68 4 achnospiraceae OTU68 Lachnospiracea OTU32 Rikenellaceae OTU38 Ruminococcaceae OTU39 Lachnospiraceae OTU102Lachnospiraceae OTU469Lachnospiraceae OTU293Lachnospiraceae OTU21 Ruminococcaceae TTU100Ruminococcaceae T) OTU13 Lachnospiraceae OTU12 Lachnospiraceae OTU11 Ruminococcaceae OTU27 Lachnospiraceae OTU520Lachnospiraceae OTU3 Lachnospiraceae oTu304I OTU24 Peptostreptococcaceae OTU24 3 Lachnospiraceae OTU37 Lachnospiraceae OTU10 Atopobiaceae OTU9 Ruminococcaceae OTU31 Lachnospiraceae OTU28 Bacteroidaceae OTU5 Desulfovibrionaceae OTU47 Ruminococcaceae OTU20 Tannerellaceae OTU93 Ruminococcaceae OTU1 Desulfovibrionaceae OTU43 Veillonellaceae OTU534Bacteroidaceae OTU19 Bacteroidaceae oTU34 Ruminococcaceae

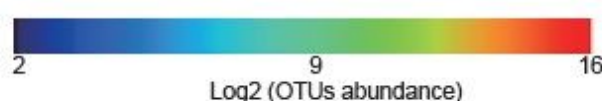

Log2 (OTUs abundance)

\section{Figure 1}

Effect of dietary yeast culture supplementation on bacterial community composition in goose gut.

Number of shared OTUs among five groups (a); heat map of changes in relative abundance of the 50 most abundant OTUs summarized, at the family level (b); composition of shared gut microbiota in the five groups, at the phylum level (c); relative abundance of gut microbiota that is uniquely present in the YC group, at the genus level (d). 
a)

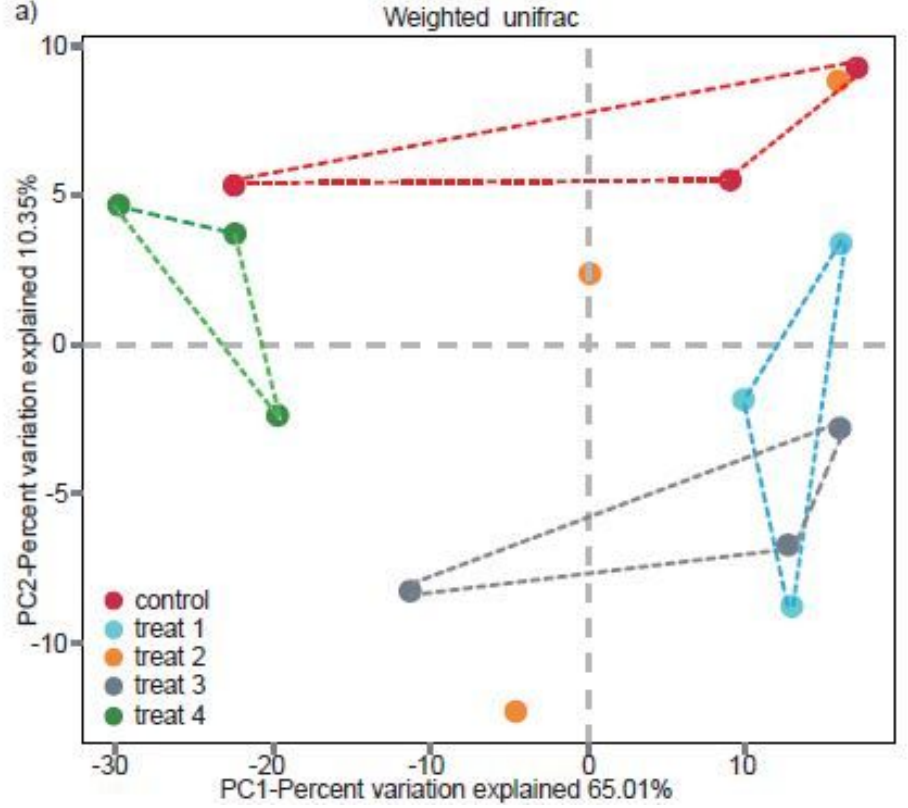

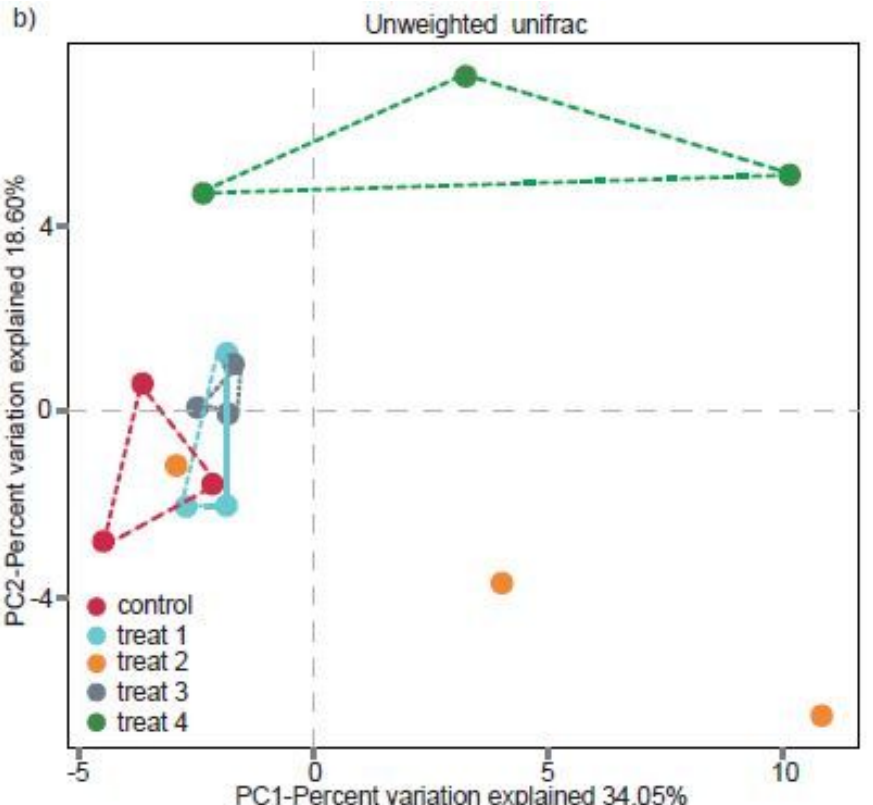

\section{Figure 2}

Effect of dietary yeast culture supplementation on microbiota community structure in goose gut. Principal coordinates analysis (PCoA) using weighted (a) or unweighted (b) UniFrac distances.

a)

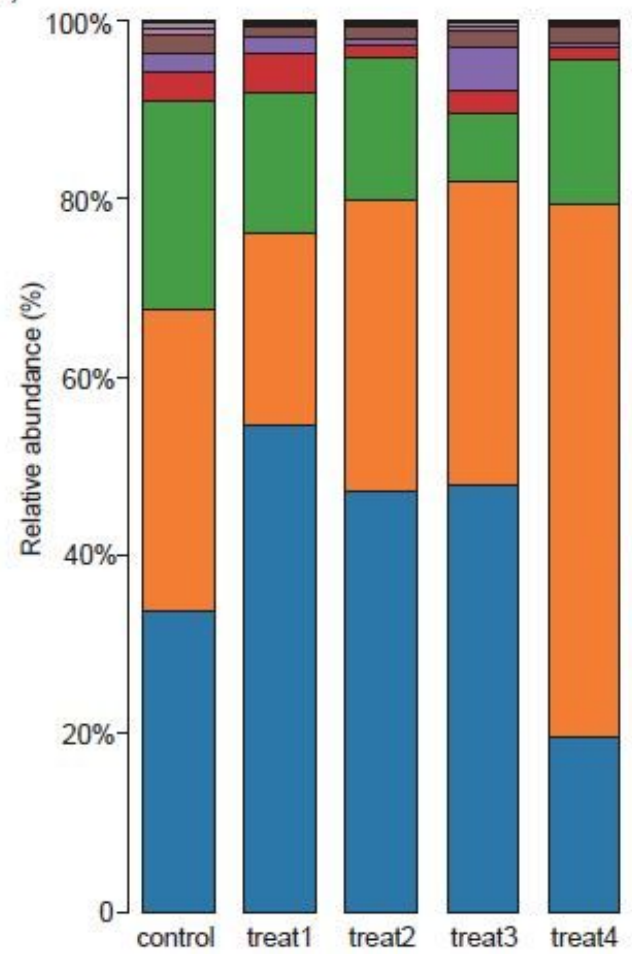

b)

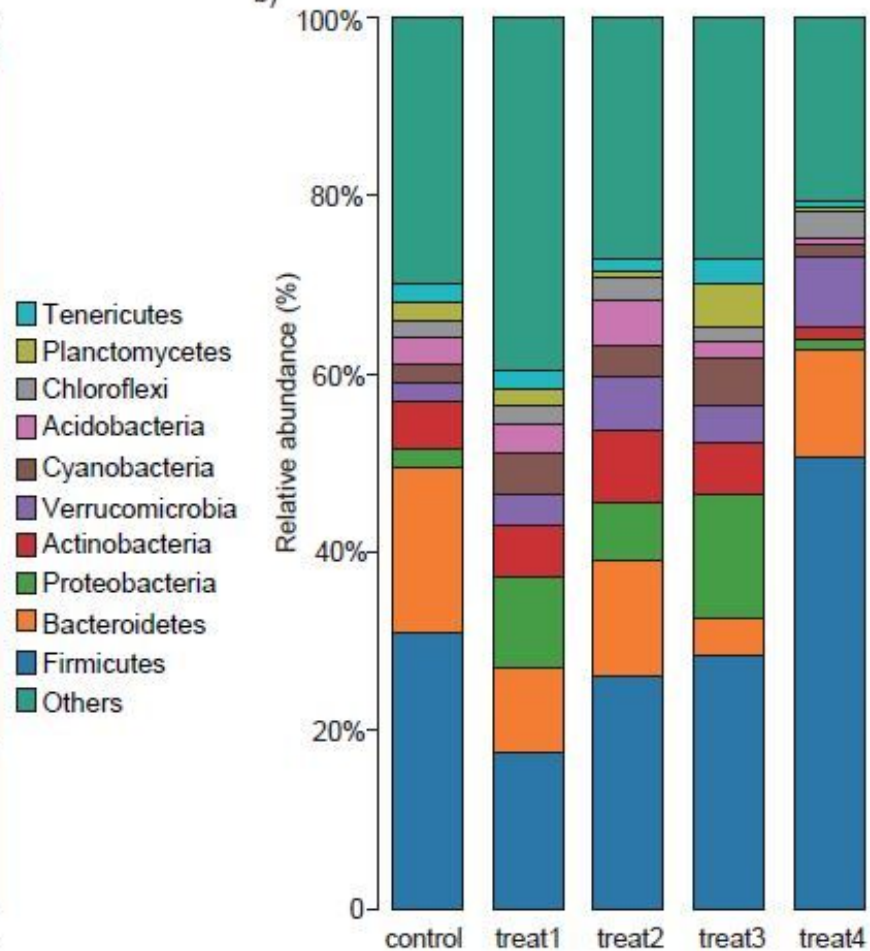

$\square[$ Ruminococcus]_torques_group

$\square$ Akkermansia

$\square$ Ruminococc

$\square$ Subdoligranulum

$\square$ Blautia

$\square$ Bacteroides

$\square$ Lachnospiraceae

$\square \mathrm{CHKCI001}$

$\square$ Desulfovibrionaceae

$\square$ Alistipes

$\square$ Others

\section{Figure 3}

Effect of dietary yeast culture supplementation on the relative abundance of gut microbial population in geese. Relative abundance of bacterial phyla (a) and genera (b). 

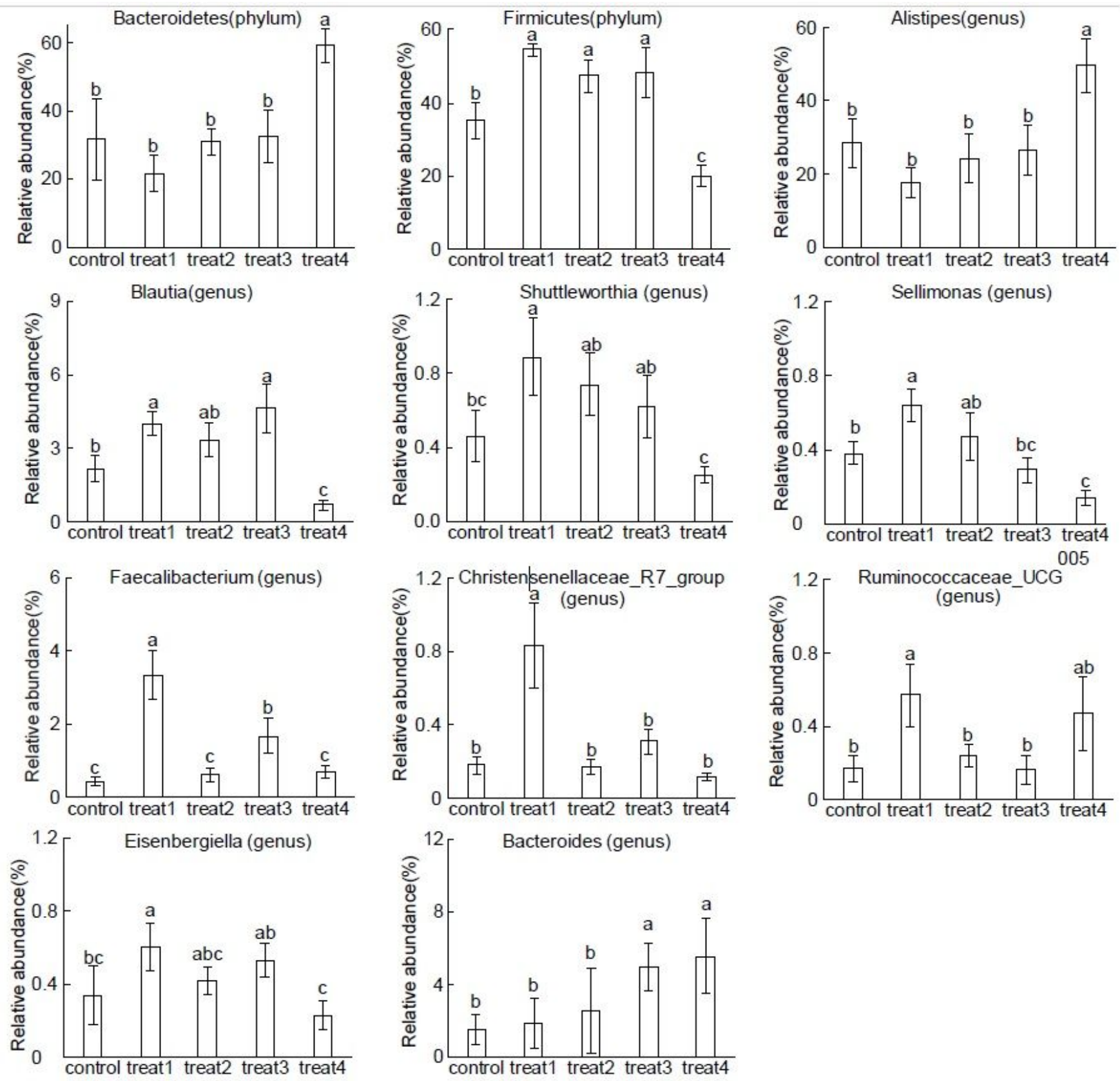

Figure 4

Effect of dietary yeast culture supplementation on relative abundance of gut bacteria in geese which were significantly affected by YC treatment. 
a)

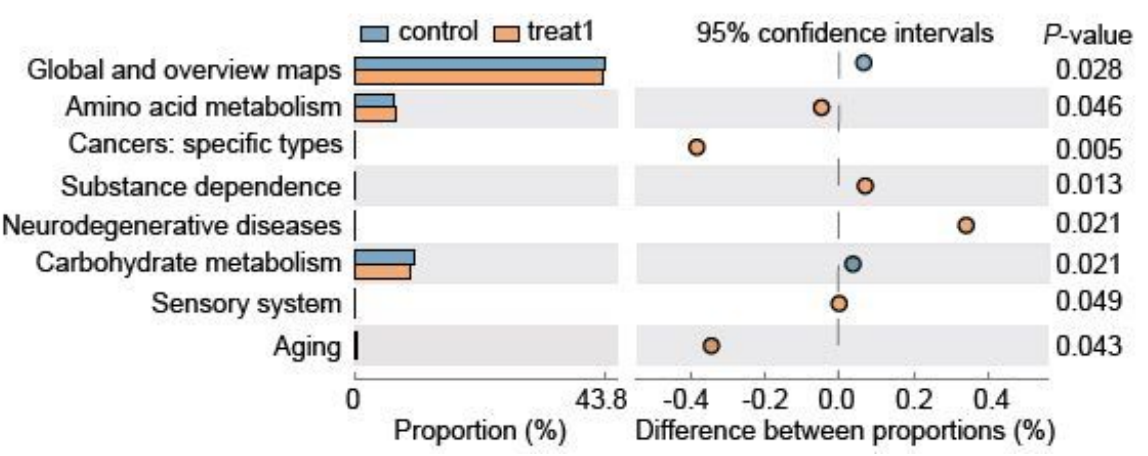

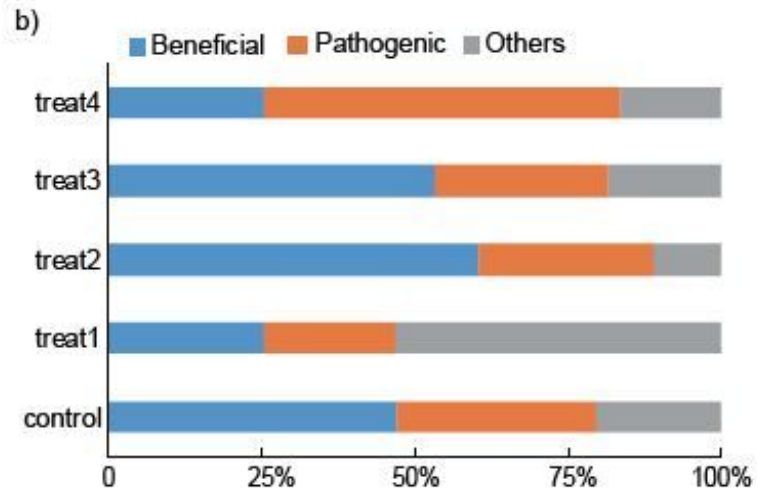

\section{Figure 5}

Effect of YC dietary yeast culture supplementation on the function of gut bacteria in geese. Predicted function of gut microbiota (a); proportion of potential beneficial and pathogenic bacteria at the genus level (b).

\section{Supplementary Files}

This is a list of supplementary files associated with this preprint. Click to download.

- SupplementaryInformation.docx 